\title{
Hermit crab response to a visual threat is sensitive to looming cues
}

\author{
Talya Shragai $^{1}{ }^{\text {, }}$ Xiaoge Ping ${ }^{2}$, Cameron Arakaki ${ }^{3}$, Dennis Garlick ${ }^{3}$, Daniel T Blumstein ${ }^{1}$, Aaron P Blaisdell \\ Corresp. 3 \\ 1 \\ Department of Ecology and Evolutionary Biology, University of California, Los Angeles, Los Angeles, CA, United States \\ 2 Key Laboratory of Animal Ecology and Conservation Biology, Institute of Zoology, Chinese Academy of Sciences, Beijing, China \\ 3 Department of Psychology, University of California, Los Angeles, Los Angeles, CA, United States \\ Corresponding Author: Aaron P Blaisdell \\ Email address: blaisdell@psych.ucla.edu
}

Prior work in our lab has shown that an expanding image on a computer screen elicits a hiding response in the Caribbean terrestrial hermit crabs (Coenobita clypeatus). We conducted two experiments to identify what properties of the expanding stimulus contribute to its effectiveness as a visual threat. First we found that an expanding geometric star evoked a strong hiding response while a contracting or full-sized stationary star did not. A second experiment revealed that the more quickly the stimulus expanded the shorter the latency to hide. These findings suggest that the anti-predator response to looming stimulus relies heavily on visual cues relating to the manner of approach. The simulated visual threat on a computer screen captures key features of a real looming object that elicits hiding behavior in crabs in the wild. 
1 Hermit crab response to a visual threat is sensitive to looming cues

2

3 Talya Shragai • Xiaoge Ping • Cameron Arakaki • Dennis Garlick • Daniel T. Blumstein

4 - Aaron P. Blaisdell

5

6 T. Shragai • D.T. Blumstein

7 Department of Ecology and Evolutionary Biology, University of California, 621 Young Drive

8 South, Los Angeles, CA 90095-1606, USA

9 E-mail: $\underline{\text { marmots@ucla.edu }}$

10

11 X. Ping - Key Laboratory of Animal Ecology and Conservation Biology, Institute of Zoology,

12 Chinese Academy of Sciences, Beijing, China.

13 C. Arakaki • D. Garlick • A.P. Blaisdell

14 Department of Psychology, University of California, 1285 Franz Hall, Los Angeles, CA 90095-

151563 , USA

16 E-mail: blaisdell@psych.ucla.edu 


\section{Abstract}

22 Prior work in our lab has shown that an expanding image on a computer screen elicits a hiding

23 response in the Caribbean terrestrial hermit crabs (Coenobita clypeatus). We conducted two

24 experiments to identify what properties of the expanding stimulus contribute to its effectiveness

25 as a visual threat. First we found that an expanding geometric star evoked a strong hiding

26 response while a contracting or full-sized stationary star did not. A second experiment revealed

27 that the more quickly the stimulus expanded the shorter the latency to hide. These findings

28 suggest that the anti-predator response to looming stimulus relies heavily on visual cues relating

29 to the manner of approach. The simulated visual threat on a computer screen captures key

30 features of a real looming object that elicits hiding behavior in crabs in the wild. 


\section{Introduction}

33 Many species rely on vision for threat detection. The ability to appropriately respond to an

34 approaching predator requires the ability to detect the visual threat and estimate its imminence

35 (Fanselow \& Lester, 1988). In a natural context, rapidly approaching visual stimuli elicit the

36 looming response (Chan et al., 2010a). It can be challenging to experimentally manipulate

37 individual features of the looming stimulus to determine their effectiveness. By using a looming

38 image on a computer screen, it is possible to simulate an approaching predator in controlled

39 laboratory conditions and measure the nature and timing of responses. Using such methods,

40 many species have been shown to flee or hide from looming images (e.g., Chan et al., 2010b;

41 Fotowat \& Gabbiani, 2011; Preuss et al., 2006; Rind \& Simmons, 1999; Sun \& Frost, 1998, \&

42 Yilmaz \& Meister, 2013).

43 But not all looming images are associated with an increased predation risk. Thus, other

44 studies have manipulated the image content to determine whether a variety of images are

45 similarly evocative. For instance, Curio (1974) manipulated the structure of a looming predator

46 image to show that pied flycatchers (Ficedula hypoleuca) used a complex combination of visual

47 cues to discriminate both between predators and non-predators, and between similar types of

48 predators. Similar discriminative abilities have been reported from observational field

49 experiments. For instance, Walters (1990) showed that three species of lapwings (Charadriidae:

50 Vanellus spp.) also use visual cues to identify predators while Seyfarth and Cheney (1990)

51 demonstrated that vervet monkeys (Chlorocebus pygerythrus) emitted different alarm calls in

52 response to the sight of different predators (see Yilmaz \& Meister, 2013, for similar results in

53 mice).

54 Species are also sensitive to the rate at which an object expands because rate contains 
55 potential information about the time to impact. Studies of crayfish (Procambarus clarkii, Glantz, 56 1974), fish (Carassius auratus, Pruess et al., 2006), and fiddler crabs (Neohelice granulate,

57 Hemmi \& Pfeil, 2010; Oliva \& Tomsic, 2012) have measured response differentiation when the 58 speed or size of the looming image was changed, showing that species are sensitive to alter the 59 timing of their response based on calculations of the timing of collision.

60 Caribbean terrestrial hermit crabs (Coenobita clypeatus) are an ideal invertebrate system to 61 test looming image discrimination and processing. Prior work in our lab demonstrated that

62 hermit crabs have a binary escape response; they hide (or fail to hide) in their shells when

63 presented with a looming image of a predator or geometric shape presented on a computer screen

64 (Chan et al. 2010b; Ping et al., 2015; Ryan et al. 2012; Stahlman et al. 2011; Watanabe et al. 65 2012).

66 Using procedures developed in our lab, we designed two experiments to manipulate specific

67 aspects of the image and then measured responses by recording the number of trials to

68 habituation, the latency to hide, and the latency to emerge from their shells. The goal of the

69 experiments was to determine which factors contributed to the effectiveness of the looming

70 visual stimuli used in our prior research to elicit antipredator hiding responses. The first

71 experiment compared the typical looming stimulus produced by the gradual expansion of the

72 image with two other conditions. In one condition, the stimulus was presented suddenly at full

73 size, and in the other the stimulus began at full size and contracted to a single pixel. The second

74 experiment tested how changing the rate of image expansion affected response times.

\section{General Methods}

76 The subjects were 57 hermit crabs acquired from a pet supply company (California

77 Zoological Supply). Experimentally-naïve crabs were used for each experiment. The hermit 
78 crabs' shells aperture length ranged from 3 to $4 \mathrm{~cm}$ across. Crabs were housed in UCLA's

79 Comparative Cognition Laboratory (http://pigeonrat.psych.ucla.edu) in groups of one to six.

80 Each had its claw numbered with non-toxic OPI nail polish for identification. Subjects were

81 housed in $50 \times 25 \times 25 \mathrm{~cm}$ clear plastic bins. Bins were lined with coconut fiber substrate (Zoo

82 Med Eco Earth, San Luis Obispo, CA, USA) and each contained two ceramic water dishes, a

83 paper plate under which the crabs could comfortably rest, a wet sponge to maintain humidity,

84 and a plastic food cup. Crabs always had access to distilled drinking water in one dish and a $1 \%$

85 salt solution in the other, and were given Tetrafauna Hermit Crab meal pellets daily (Blacksburg,

86 VA, USA). The coconut substrate was checked daily for fungus and changed three times a week

87 or more often as necessary to prevent infection. The dishes containing water were cleaned and

88 replenished daily, and the coconut substrate was replaced on Mondays, Wednesdays, and

89 Fridays. Each bin was mostly covered with a clear acrylic sheets $(0.5 \mathrm{~cm}$ thick) to maintain

90 humidity, with a small opening to allow air circulation. The room contained a Vicks humidifier

91 (Proctor \& Gamble, Cincinnati, OH, USA) to maintain the atmosphere at 50-75\% humidity in

92 the bins. Two 9.5 Watt heat lamps were used to provide warmth for the bins, maintaining a room

93 temperature of $25^{\circ} \mathrm{C}$. A timer provided a 14:10 hour light: dark cycle, with lights turning on at

94 8:00 AM.

95 All experimental trials were performed in a test arena located on a desktop (Fig. 1). We

96 constructed a wooden, matt black arena $18.2 \mathrm{~cm} \mathrm{~W} \mathrm{x} 30.5 \mathrm{~cm} \mathrm{~L}$, x $14.5 \mathrm{~cm} \mathrm{H})$. A 15 -inch LCD

97 monitor (Dell 1704FPVt, Round Rock, TX, USA) with a screen resolution of 1024X 768 pixels

98 was used to display visual stimuli. Subjects were secured in a crab-holder using Fun $\mathrm{Tak}^{\mathrm{TM}}$ blue

99 and white putty (Henkel Removable Poster Putty ${ }^{\mathrm{TM}}$, model 1084014, Duseldorf, Germany)

100 attached to the crab's shell. The holder was positioned in front of and $13.3 \mathrm{~cm}$ away from the 
101 LCD screen. Subjects were oriented so that the aperture of their shell was angled up and

102 perpendicular in relation to the ground when secured.

103 We used a custom program developed in-house to automatically recorded latency to hide and 104 re-emerge (Ryan et al., 2012). A web camera (Logitech, model C250, Lausanne, Switzerland) 105 was placed $5 \mathrm{~cm}$ in front of the clamp, below the LCD monitor. The program detected activity in 106 a $300 \times 150$ pixel region in the camera's visual field. At the beginning of a session, the subject 107 was placed on its back into the putty and clipped into the apparatus. The square-shaped visual 108 detection field was positioned so that the bottom $1 / 3^{\text {rd }}$ contained the crab's shell and the top $2 / 3^{\text {rds }}$ 109 was empty to allow detection space for when the crab emerged. We then set the number of 110 "hiding pixels" (i.e., the number of pixels the detector registered when the crab was hiding), and 111 the program computed a value to set a variable called "ExtraPixelsOut" to determine when the 112 crab had emerged from its shell. The value of "ExtraPixelsOut" was calibrated for each 113 individual crab at the beginning of each session to account for each individual crab's size.

114 Furthermore, to account for variation in individual size, we calculated a variable labeled "ClawSize" for each crab. To calculate "ClawSize" we set the baseline for a hiding crab at zero 116 pixels, recorded the minimal number of pixels once the crab emerged, subtracted 1000 from this 117 number, and divided it by 0.5 . After this calibration was complete, a crab was scored as hiding 118 until this additional number of pixels was detected in the recording square on the monitor. We 119 found this method to reliably discriminate the state of the crab being emerged from its shell from 120 the state of it hiding within its shell (Ryan et al., 2012).

121 A variable labeled "ExtraPixelsIn" was computed to ensure the program accurately registered 122 when the crab returned to hiding. After emerging from its shell, a hiding response did not always 123 result in the crab returning as far into its shell as when the initial "hiding pixels" baseline was set 
124 at the beginning of the session. Thus, to increase sensitivity to detecting hiding responses,

125 "ExtraPixelsIn" was set to $1 / 6^{\text {th }}$ the hiding pixels. Once the crab emerged, the program did not 126 consider the crab as being "hiding" until the number of pixels detected fell below this number of 127 additional pixels (Ryan et al., 2012).

128 The criterion for number of trials to habituation was defined as two consecutive trials with no 129 registered hide response. Both experiments were video recorded and the results of the automatic 130 detector were verified on the video and hand scored when necessary to include cases where the 131 automatic detector failed to detect response.

132 If a subject failed to emerge for $20 \mathrm{~min}$ at the beginning of a session it was returned to its 133 home cage and was tried again after all the other crabs had completed their session for the day. If 134 a hermit crab still failed to emerge from its shell, it was not included in the experiment. 135 Experiment 1

136 In this experiment we compared the effectiveness of an expanding stimulus, a contracting 137 stimulus, and a full-sized stationary stimulus on eliciting the hide response.

138 Methods

139 Subjects. We tested 30 experimentally-naïve crabs. 10 crabs were tested on day 1, another 10 140 were tested on day 2, and the remaining 10 crabs were tested on day 3 of the experiment. Each 141 day, about $1 / 3^{\text {rd }}$ of the crabs were tested in each of the three conditions: Expanding, Contracting, 142 and Static. Test order was counterbalanced using a Latin square design to account for 143 confounding effects of time of day and day of the week.

144 Stimuli. The stimulus consisted of a white star shape, 1024 x1024 pixels, on a black background 145 (Fig. 2a).

146 Procedure. A session started with the crab being placed in the apparatus as described in the 
147 General Methods. Each trial began as soon as the crab was automatically detected as being out of

148 its shell for 30 consecutive seconds. If the program detected that the crab hid again during this

149 interval, the pre-trial timer would reset and not restart until the crab was detected as being "out"

150 again. Once the crab had been detected as "out" for 30 consecutive seconds, the trial began with

151 a 30-s delay followed by the presentation of the visual stimulus. Figure 3 provides a schematic

152 for the time course of each of the three types of presentation of the stimulus. The Expanding

153 image started as a single pixel at the top of the screen and enlarged at an exponentially increasing

154 rate such that it simulated the approach of the stimulus at a constant rate. From the start

155 presentation, the stimulus reached its full width of approximately 900 pixels in $17 \mathrm{~s}$. Once the

156 stimulus reached its largest size, it filled the lower part of the screen and remained on the screen

157 for an additional $5 \mathrm{~s}$. For Static presentations, the stimulus was presented at full size at the center

158 of the screen for $22 \mathrm{~s}$. For Contracting presentations, the stimulus was initially presented at its

159 full size and contracted at an exponentially decreasing rate to simulate the image receding at a

160 constant rate. The contracting presentation took $17 \mathrm{~s}$ to go from full size to a single pixel at the

161 top of the screen, after which the single pixel remained on the screen for an additional $5 \mathrm{~s}$.

162 For all conditions, trials were repeated until either the visual stimulus had been presented

16315 times, the session reached a time ceiling of $30 \mathrm{~min}$, or until the crab had two consecutive trials

164 on which it did not hide at all during the stimulus presentation (i.e., reached the habituation 165 criterion).

166 Results

167 Very few subjects in the Contracting or Static conditions hid to the stimulus presentation 168 on any trial, precluding a group comparison of rates of habituation. Therefore, we instead 169 analyzed proportion of crabs that hid on the first trial in each of the three conditions using a two- 
170 tailed Freeman-Halton extension of Fisher's exact test. For all tests, we interpret two-tailed $p$ -

171 values $<0.05$ as significant.

172 Stimulus presentation had a large effect on hiding responses $(p=0.023$; Fig. 4$)$, with the

173 Expanding stimulus eliciting the most reliable response. A larger proportion of crabs in the

174 Expanding condition hid as compared to the Static or Contracting conditions (Fisher's exact test:

$175 p=0.023)$. There was no difference in the proportion of crabs that had in the Static or

176 Contracting conditions $(p=1.000)$. These results suggest that the expanding stimulus was

177 perceived as much more threatening than either the static or contracting stimuli.

178 Experiment 2

179 In this experiment we evaluated the effect of varying rate of stimulus expansion on 180 probability and latency to hide. Rate of expansion correlates with the changes in the amount of 181 stimulus that fills the visual field of the perceiver of a real looming object. Thus, to the degree

182 that rate of approach of a looming object elicit differences in latency and probability of a hiding 183 response, similar effects should be observed when rate of expansion of a two-dimensional

184 stimulus on a computer screen is varied.

185 Methods

186 Subjects. We tested 26 experimentally-naïve crabs randomly allocated to one of three groups 187 ( $n=8$ for Group 8s and 32s, and $n=10$ for Group 16s). Subjects were maintained as in Experiment 1881.

189 Stimuli. To assess the generality of the effect of expansion, two stimuli were used; an image of a 190 red-tailed hawk (Buteo jamaicensis, 1024x796 pixels at full size) and the image of a coconut 191 crab (Birgus latro, 1024x430 pixels at full size, Fig. 2b, c). All other aspects of the apparatus 192 were as described for Experiment 1. 
193 Procedure. Each subject received one session per day for two days. Each crab received one

194 session with the hawk stimulus and one session with the coconut crab stimulus, with test order

195 counterbalanced across subjects such that half of each group was shown the hawk on the first day

196 and the coconut crab on the second day while the order was reversed for the remaining crabs.

197 Groups differed in the rate of stimulus expansion from onset to reaching full size. The three

198 expansion rates tested were $8 \mathrm{~s}$ (fast velocity), $16 \mathrm{~s}$ (medium velocity), and $32 \mathrm{~s}$ (slow velocity).

199 Expansions occurred exponentially such that, in each condition, the stimulus appeared to

200 approach at a constant rate throughout the presentation.

201 Each session began and trials were conducted as described for Experiment 1. Each trial

202 consisted of the expansion from a single pixel to a full size image of either a coconut crab picture

203 or a hawk picture. Trials with the same visual stimulus were repeated until either the visual

204 stimulus had been presented 15 times, the session reached a time ceiling of $30 \mathrm{~min}$, or until the

205 crab had two consecutive trials on which it did not hide at all during the stimulus presentation

206 (i.e., reached the habituation criterion).

207 Results

208 A two-way mixed ANOVA conducted on latencies to hide on the first trial with

209 Expansion Rate (8s, 16s, and 32s) as a between-subject factor and Stimulus Type (Hawk and

210 Coconut $\mathrm{Crab}$ ) as a repeated measure revealed a main effect of Expansion Rate $F(1,23)=7.05$,

$211 p<0.01, \eta p^{2}=0.38$, but no effect of Stimulus Type, $F(1,23)=1.06, p=0.31$, nor interaction

212 between Expansion Rate and Stimulus Type, $F<1$.0. Planned comparisons revealed hide

213 latencies differed between the $8 \mathrm{~s}$ and $32 \mathrm{~s}$ presentation times $F(1,23)=13.24, p=0.01$, and

214 between the 16s and 32s presentation times $F(1,23)=7.29, p<0.05$ (Fig. 5), but not between the

$2158 \mathrm{~s}$ and $16 \mathrm{~s}$ presentation times, $F(1,23)=1.29, p=.27$. A similar ANOVA conducted on 
216 latencies to emerge failed to reveal any main effects or interactions, $F \mathrm{~s}(1,22)<1.58, p \mathrm{~s}>0.23$

217 (latency to emerge data were missing from one subject in Group 16s, and thus $n=23$ for this

218 analysis). Because a large number of subjects failed to habituate, trials to habituation was not

219 analyzed as a measure of reaction (Figure 6). These results suggest that rate of expansion serves

220 as a proxy for rate of approach of a looming stimulus, with faster rates eliciting shorter latencies

221 to hide.

\section{Discussion}

223 An expanding image was much more effective than a static or contracting image at eliciting

224 the anti-predatory hide response in hermit crabs. This supported the hypothesis that hermit crabs

225 perceived the expanding image as threatening and probably as a looming object. Moreover, the

226 faster the rate of expansion, the shorter the latency to hide, which is also consistent with how

227 animals react to looming stimuli under natural conditions.

228 Collectively, these experiments demonstrate that for Caribbean terrestrial hermit crabs, the

229 visual system plays an essential role in identifying and responding to potential threats. While

230 dynamic features of an expanding stimulus likely simulate the key aspects of a looming stimulus,

231 it is difficult to determine whether crabs perceived the pictorial or representational aspects of the

232 images. Many species show mixed evidence of perceiving photographs or pictures presented on a

233 computer screen as representations of real 3D objects versus as only 2D colored patterns. For

234 example, evidence is mixed as to whether pigeons (Columba livia) can perceive the pictorial or

235 representational aspects of pictorial displays (Fagot, 2000). We've found evidence that the

236 Caribbean hermit crab lacks color vision (Ping et al., 2015). Unpublished studies from our lab

237 also fail to find evidence that hermit crabs can distinguish between pictorial content that is

238 naturalistic (e.g., photographs of a hawk, coconut crab, sea gull), man-made real objects (e.g., a 
239 picture of a couch), and simple geometric shapes (e.g., a square, star, or oval). More research

240 would be needed to definitively answer this question for the Caribbean hermit crab.

241 Nevertheless, the fact that hermit crabs were particularly sensitive to an expanding stimulus

242 suggests that some aspects of real, looming objects are perceived in the dynamic 2D stimuli in

243 our study. Out results are consistent with much prior work exploring the neural basis of

244 antipredator responses to looming stimuli in arthropods (Hemi, 2005; Oliva et al., 2007; Oliva \&

245 Tomsic 2012).

246 While we documented the visual detection abilities that these crabs possessed, previous

247 experiments have shown the importance of the acoustic environment to the crab's ability to

248 respond to visual stimuli (Chan et al. 2010b; Ryan et al. 2012), indicating that hermit crabs use

249 multisensory channels to evaluate their environment. This is consistent with research on other

250 invertebrates such as fiddler crabs (Uca vomeris) that employ multiple detector systems to

251 evaluate different aspects of a possible threat (Hemmi, 2005; Hemmi \& Pfeil, 2010). Future

252 studies would profitably examine how assessment works in a multi-modal paradigm. Finally, it is

253 worth noting that this and prior published work from our lab demonstrates the value of going

254 from field research into the lab and back again. Field research is valuable for its ability to gather

255 questions about an organism's behaviors in its natural habitat. Laboratory research can then

256 provide a more controlled setting within which to untangle distinct behavioral processes.

\section{Conclusion}

258 Computer technology can provide a valuable tool for investigating the perceptual and 259 cognitive processes that contribute to natural behavior observed in the field. Knowledge gathered

260 from laboratory work can in turn inform new directions for field work, including tailoring

261 improvements that protect animals from potentially deleterious anthropogenic effects. 
262

263 Acknowledgements Special thanks to Hwee Cheei Lim for her constant support and

264 encouragement.

265

266 Ethical approval

267 All applicable international, national, and/or institutional guidelines for the care and use of 268 animals were followed. All procedures performed in studies involving animals were in 269 accordance with the ethical standards of the institution or practice at which the studies were 270 conducted.

271

272 References

273 Chan A. A. Y-H., Giraldo-Perez, P., Smith, S., \& Blumstein, D. T. (2010a). Anthropogenic noise 274 affects risk assessment and attention: the distracted prey hypothesis. Biol Lett 6, 458-461. 275 Chan, A. Y-H., Stahlman, W. D., Garlick, D., Fast, C. D., Blumstein, D. T., \& Blaisdell, A. P. 276 (2010). Increased amplitude and duration of acoustic stimuli enhance distraction. Animal 277 Behaviour, 80, 1075-1079.

278 Curio, E. (1975). The functional organization of anti-predator behaviour in the pied flycatcher: A 279 study of avian visual perception. Anim Behav, 23, 1-115.

280 Fagot, J. (2000) Picture perception in animals. Psychology Press: East Sussex, UK.

281 Fanselow, M. S., \& Lester, L. S. (1988). A functional behavioristic approach to aversively 282 motivated behavior: Predatory imminence as a determinant of the topography of defensive 283 behavior. In: Bolles, R.C., Beecher, M.D. (Eds.), Evolution and Learning. Lawrence Erlbaum 284 Associates, Inc., Hillsdale, NJ, pp. 185-212. 
285 Fotowat, H., \& Gabbiani, F. (2011). Collision detection as a model for sensory-motor 286 integration. Annu Rev Neurosci, 34, 1-19.

287 Glantz, R. M. (1974). Defense reflex and motion detector responsiveness to approaching targets:

288 The motion detector trigger to the defense reflex pathway. J Comp Physiol, 95, 297-314.

289 Hemmi, J. M. (2005). Predator avoidance in fiddler crabs: 2. The visual cues. Animal Behaviour, $290 \quad 69(3), 615-625$.

291 Hemmi, J. M., \& Pfeil, A. (2010). A multi-stage anti-predator response increases information on 292 predation risk. J Exp Biol, 213, 1484-1489.

293 Oliva, D., Medan, V., \& Tomsic, D. (2007). Escape behavior and neuronal responses to looming 294 stimuli in the crab Chasmagnathus granulatus (Decapoda: Grapsidae). J Exp Biol, 210, 865295880.

296 Oliva, D., \& Tomsic, D. (2012). Viso-motor transformations involved in the escape response to 297 looming stimuli in the crab Neohelice (= Chasmagnathus) granulata. J Exp Biol, 215, 3488$298 \quad 3500$.

299 Ping X., Lee J. S., Garlick D., Jiang Z., \& Blaisdell A. P. (2015). Behavioral evidence

300 illuminating the visual abilities of the terrestrial Caribbean hermit crab, Coenobita clypeatus. 301 Behavioural Processes, 118, 47-58.

302 Preuss, T, Osei-Bonsu, P. E., Weiss, S. A., Wang, C., \& Faber, D. S. (2006). Neural 303 representation of object approach in a decision-making motor circuit. J Neurosci, 26, 34543043464.

305 Rind, F. C., \& Simmons, P. J. (1999). Seeing what is coming: building collision-sensitive 306 neurons. Trends Neurosci, 22, 215-220 
307 Ryan, K. M., Blumstein, D. T., Blaisdell, A. P., \& Stahlman, W. D. (2012). Stimulus

308 concordance and risk-assessment in hermit crabs (Coenobita clypeatus): Implications for 309 attention. Behav Process, 91, 26-29.

310 Stahlman, W. D., Chan, A. Y-H., Blumstein, D. T., Fast, C. D., \& Blaisdell, A. P. (2011).

311 Auditory stimulation dishabituates anti-predator escape behavior in hermit crabs (Coenobita 312 clypeatus). Behav Process, 88, 7-11.

313 Seyfarth, R., \& Cheney, D. (1990). The assessment by vervet monkeys of their own and another 314 species' alarm calls. Anim Behav, 40, 754-764.

315 Sun, H., \& Frost, B. J. (1998). Computation of different optical variables of looming objects in 316 pigeon nucleus rotundus neurons. Nat Neurosci, 1, 296-303.

317 Walters, J. R. (1990). Anti-predatory behavior of lapwings: Field evidence of discriminative 318 abilities. Wilson Bull, 102, 49-70.

319 Watanabe, N. M., Stahlman, W. D., Blaisdell, A. P., Garlick, D., Fast, C. D., \& Blumstein, D. T. 320 (2012). Quantifying personality in the terrestrial hermit crab: Different measures, different 321 inferences. Behav Process, 91, 133-140.

322 Yilmaz, M. \& Meister, M. (2013). Rapid innate defensive responses of mice to looming visual 323 stimuli. Current Biology, 23, 2011-2015. 
325 Figure 1 A schematic of our experimental set-up. The monitor displays the fully enlarges image 326 of the coconut crab stimulus. Figure not drawn to scale.

327

328 Figure 2 Visual stimuli. Star image for Experiment 1 (a), Hawk and Coconut Crab images for 329 Experiment 2 (b, c). At maximum size, the Hawk image is 1024 x 796 pixels and the Coconut 330 Crab image is $1024 \times 430$ pixels.

331

332 Figure 3 Schematic of the conditions: A) expanding, B) full-sized, and C) contracting stimulus 333 for Experiment 1.

334

335 Figure 4 Percent of crabs that hid to the Expanding, Contracting, and Static conditions in 336 Experiment 1. Asterisk indicates significant difference from remaining groups, $p<.05$.

Figure 5 Mean latency (s) to hide to the $8 \mathrm{~s}, 16 \mathrm{~s}$, and 32s presentation time stimuli using both a Hawk and Coconut Crab image in Experiment 2. Error bars depict standard errors of the means.

341 Figure 6 Percentage of subjects that reached the habituation criterion in each condition of 342 Experiment 2. 
Figure 1

A schematic of our experimental set-up.

The monitor displays the fully enlarges image of the coconut crab stimulus. Figure not drawn to scale

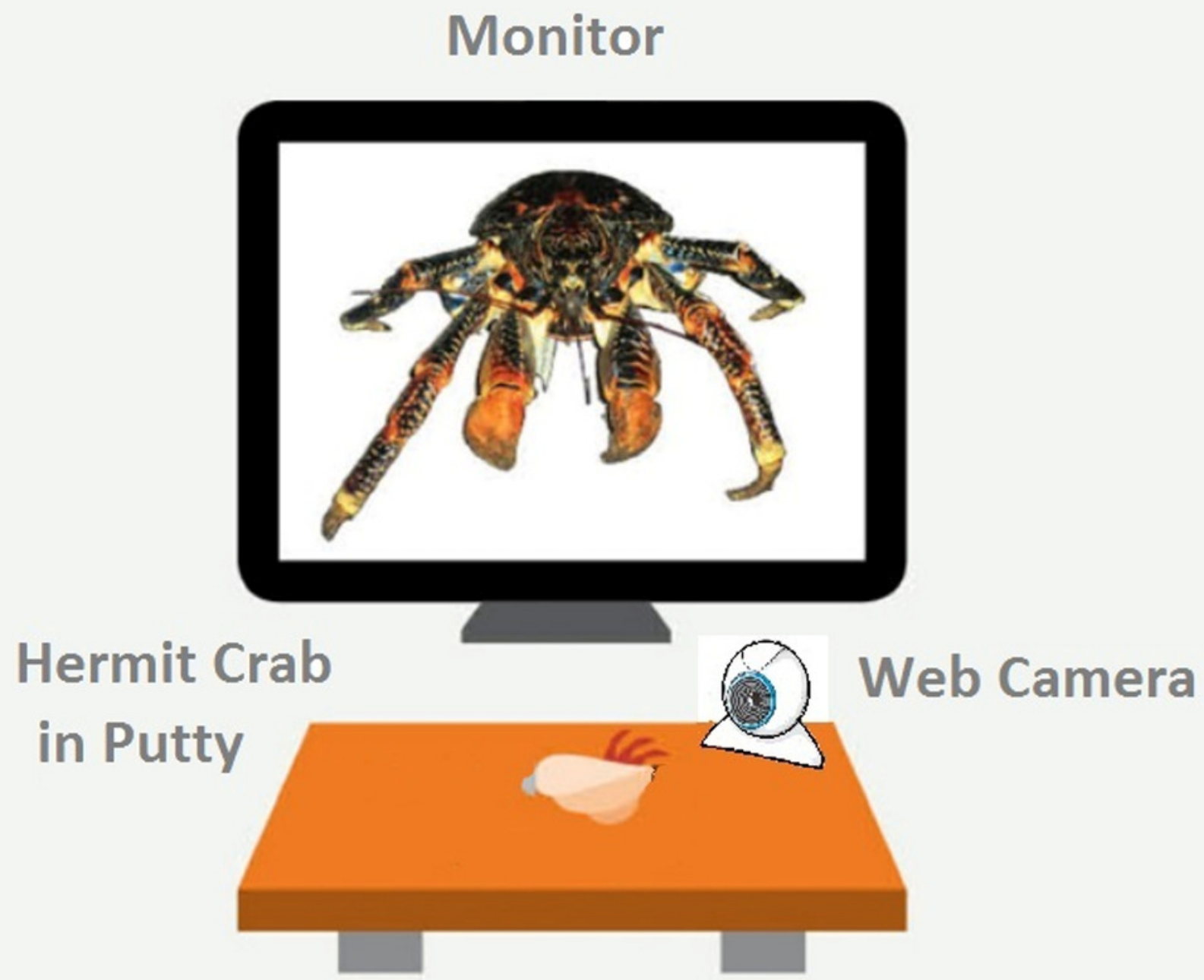


Figure 2

Visual stimuli.

Star image for Experiment 1 (a), Hawk and Coconut Crab images for Experiment 2 (b, c).

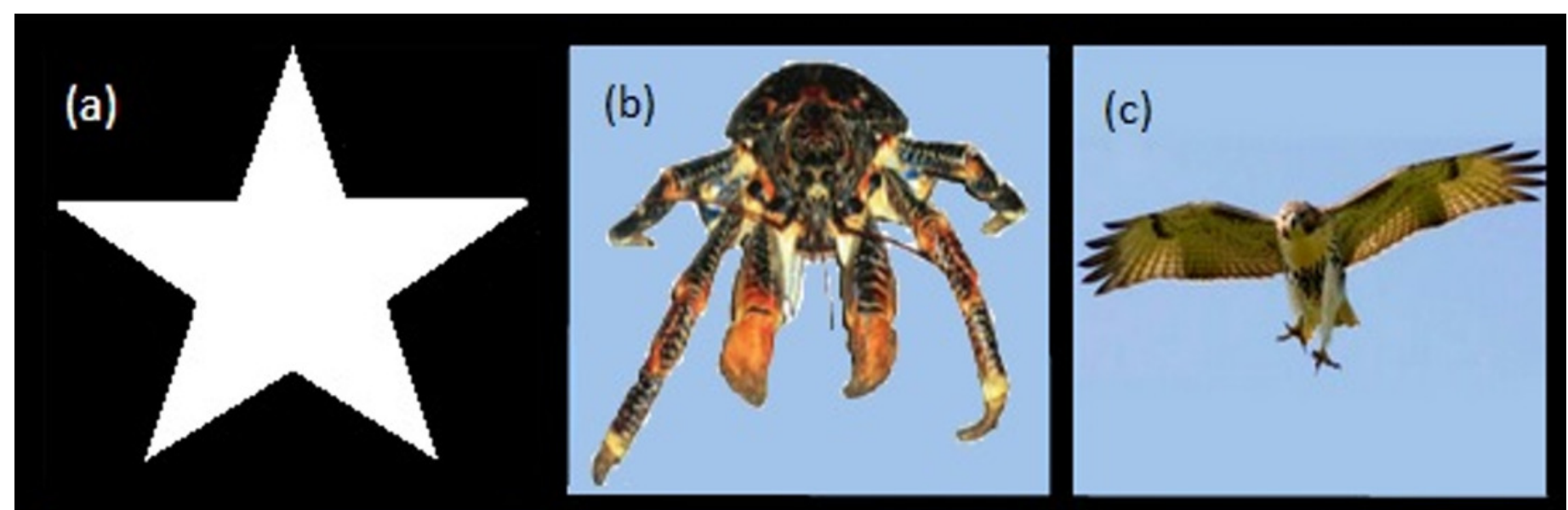


Figure 3

Presentation formats for Experiment 1

Schematic of the conditions, expanding, full-sized, and contracting stimulus for Experiment 1.
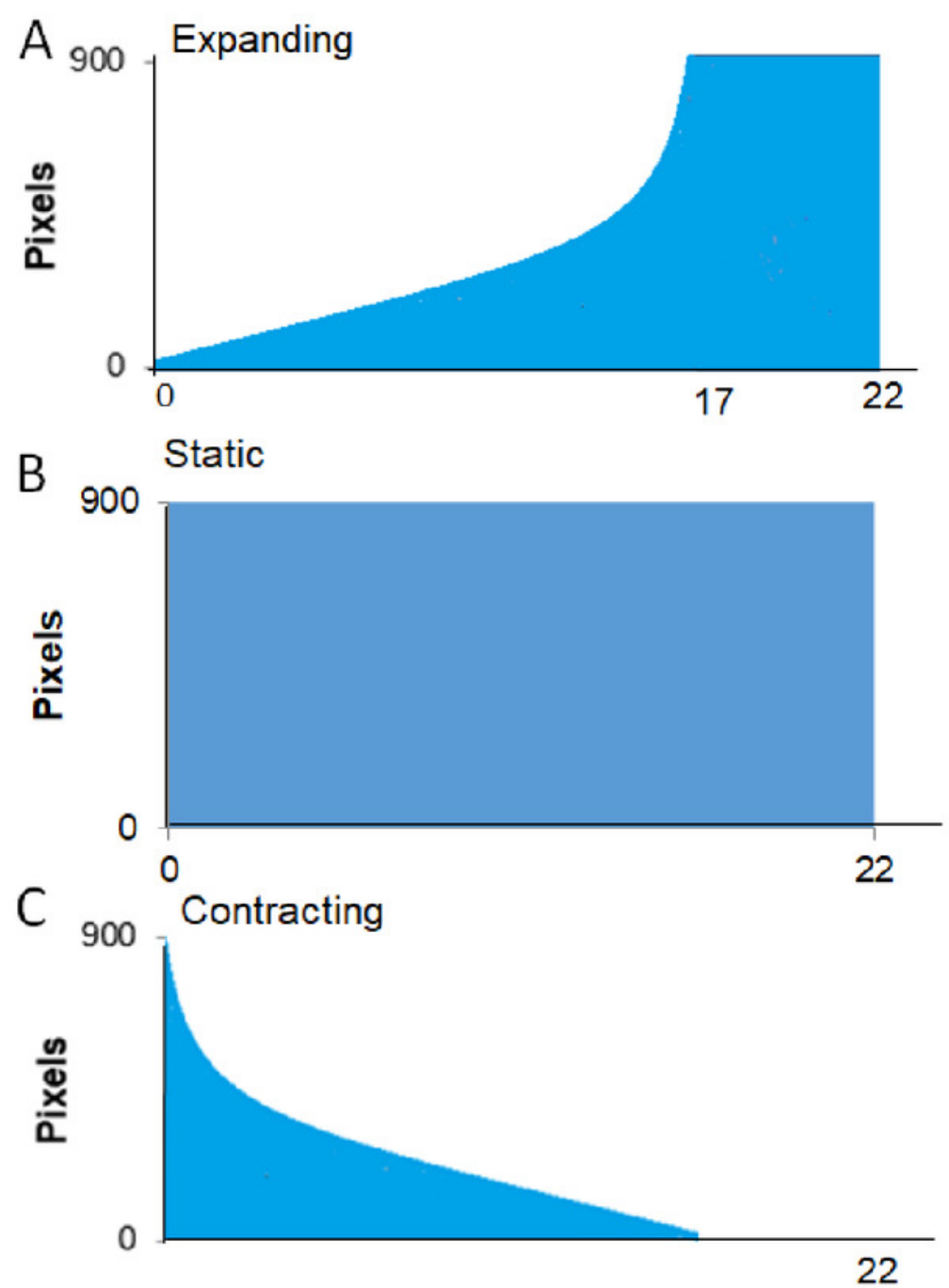
Figure 4

Percent of crabs that hid to the Expanding, Contracting, and Static conditions in Experiment 1.

Asterisk indicates significant difference from remaining groups, $p<.05$.

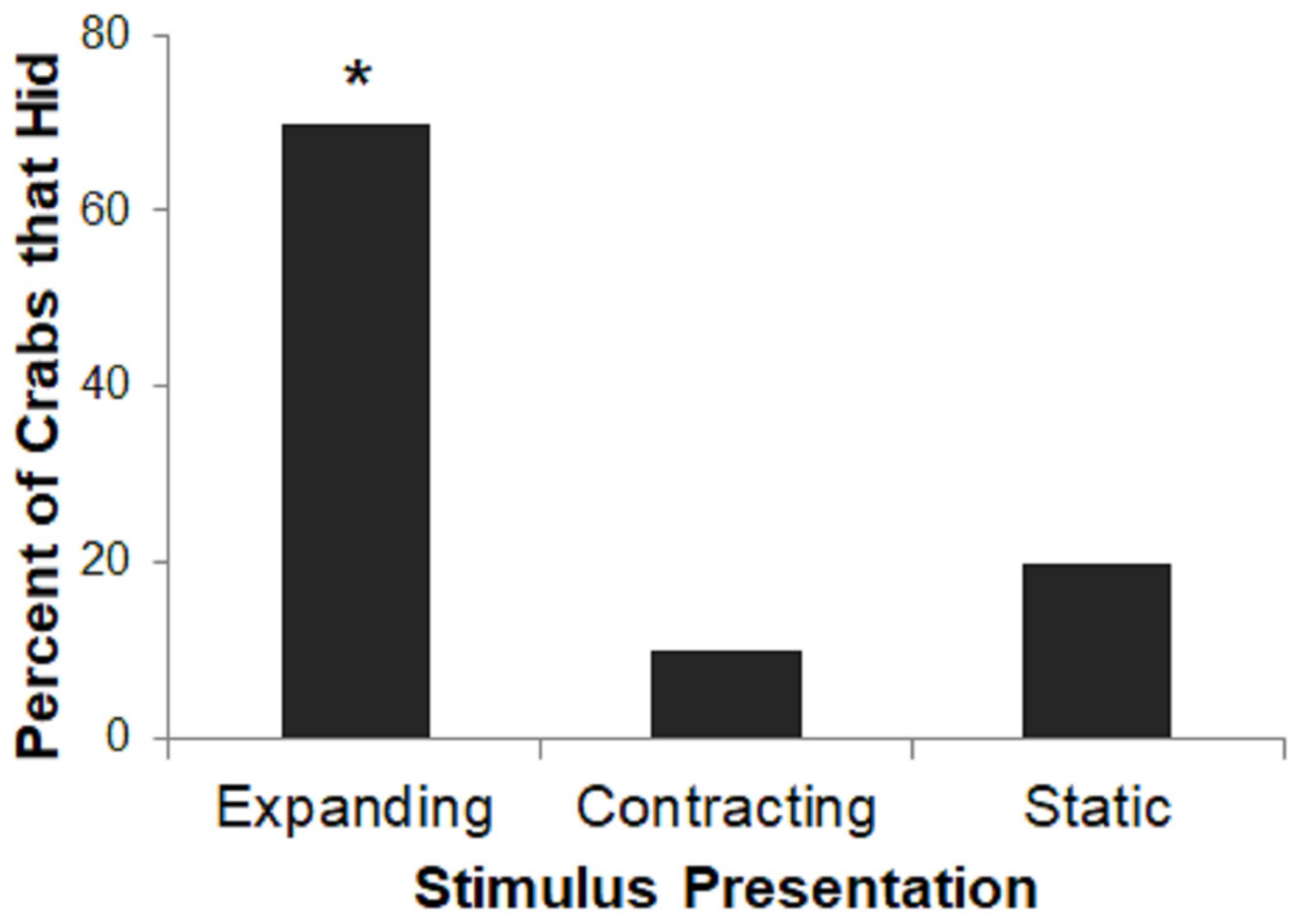


Figure 5

Results Experiment 2

Mean latency (s) to hide to the $8 \mathrm{~s}, 16 \mathrm{~s}$, and $32 \mathrm{~s}$ presentation time stimuli using both a Hawk and Coconut Crab image in Experiment 2. Error bars depict standard errors of the means.

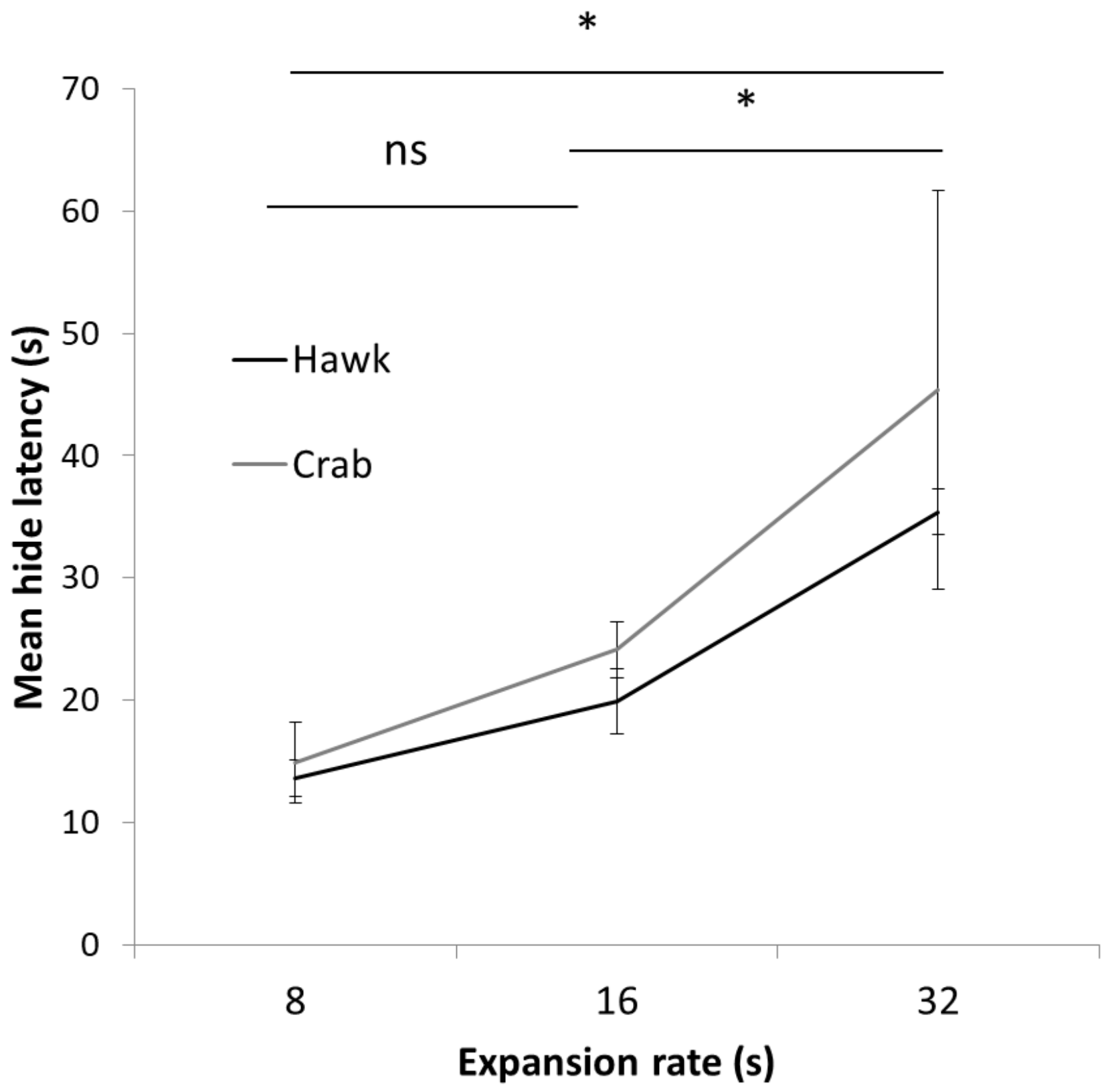


Figure 6

Percentage of subjects that reached the habituation criterion in each condition of Experiment 2.

\section{Hawk \\ Expansion rate (s)

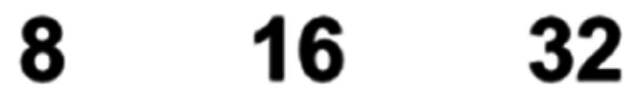 \\ $37.5 \% \quad 60 \% \quad 50 \%$ \\ $62.5 \% \quad 40 \% \quad 50 \%$}

Habituated

Not habituated 